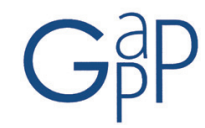

GESTIÓN Y ANÁLISIS DE POLÍTICAS PÚBLICAS, Nueva Época, nº 12 julio-diciembre 2014 ISSN: 1989-8991

DOI: http://dx.doi.org/10.24965/gapp.voi12.10211

\title{
Envejecimiento y políticas sociales integradas en la Ciudad de México
}

\author{
Kristiano Raccanello \\ Departamento de Economía, Fundación Universidad de las Américas Puebla \\ kristiano.raccanello@udlap.mx \\ Martha Isabel Ángeles Constantino \\ Universidad Autónoma del Estado de México \\ mangeles constantino@hitmail.com \\ Nallely Molina Velasco \\ Consejo de Evaluación del Desarrollo Social del Distrito Federal \\ nallely.molina@hotmail.com \\ Recibido: 30 de mayo de 2014 \\ Aceptado: 29 de julio 2014
}

\section{Resumen}

Las personas adultas mayores aun manteniendo todos sus derechos, enfrentan obstáculos dictados por su condición y por una menor integración social. Frente a la falta de un marco institucional que vele de forma explícita para este grupo etario, el Gobierno de la Ciudad de México ha promulgado leyes que constituyen un importante avance en este sentido. Este marco legal se anticipa al aumento de la población adulta mayor en la Ciudad de México en las siguientes décadas y sustenta los programas y acciones que la Secretaría de Desarrollo Social del Distrito Federal, a través del Instituto para la Atención de los Adultos Mayores en el Distrito Federal, está llevando a cabo. Tanto los programas como las acciones buscan promover la inclusión social de manera integrada para mejorar el nivel de bienestar de las personas adultas mayores.

\section{Palabras clave}

Personas adultas mayores, derechos, desarrollo social, política social, México.

\section{Aging and integrated social policies in Mexico City}

\section{Abstract}

Older people, although preserving all their rights, have to deal with obstacles caused by their status and a lesser social integration. Because an institutional framework to protect such age group is missing, the Mexico City Government has enacted several laws that constitute a remarkable advancement in this regard. This legal framework both anticipates the increasing elderly population in Mexico City and supports programs and activities that are being undertaken by the Ministry of Social Development of the Mexico City Government, through the Institute for the Care of Older Adults in Mexico City.

Both programs and activities are aimed at promoting social inclusion, as well as improving the welfare of the elderly.

\section{Keywords}

Elderly, rights, social development, social policy, Mexico. 


\section{ANTECEDENTES}

Como resultado de paulatinos procesos de investigación y desarrollo en distintas áreas del conocimiento, los avances tecnológicos se han manifestado en un mayor bienestar de la población desde el punto de vista del consumo, a veces a costa de la calidad de vida de los individuos y del medio ambiente. También, no sólo se ha proporcionado un mayor número de bienes y servicios, sino que las personas también viven por más tiempo.

Si por un lado este aspecto representa un resultado positivo para la humanidad en su conjunto, por otro se reconoce que la calidad de vida de los individuos no es uniforme al estar vinculada a factores socioeconómicos (género, edad, recursos, apoyo por parte de otros familiares, redes sociales, etc.) así como a los servicios e infraestructura disponibles y a su alcance.

De esta forma, y sin que el orden constituya una prioridad implícita en nuestro argumento, el "cuánto tiempo vivir" ha puesto una interrogante paralela: el "cómo vivir", concepto que se resume en la calidad de vida de una persona, no sólo durante su etapa productiva, sino, y sobre todo, una vez jubilada. Este planteamiento se fundamenta en la observación de que las personas adultas mayores ${ }^{1}$ frecuentemente viven con mayores restricciones desde un punto de vista económico y social. Las primeras son el resultado de las decisiones de ingreso y consumo que han sido tomadas en los años previos, mientras que las segundas responden a aspectos ligados a la sociedad en su conjunto. Por un lado, las necesidades económicas, en concomitancia con un mayor individualismo, han impulsado a privilegiar los propios intereses con una menor atención hacia los demás y, por otro, las redes sociales de las personas adultas mayores se adelgazan de forma natural con el pasar del tiempo. De esta forma, estas personas pueden verse marginadas tanto por los familiares, quienes no pueden o no quieren hacerse cargo de ellos, como por la sociedad, que no les proporciona el apoyo y la consideración que merecen. Esta situación puede ser el resultado del desconocimiento por parte de la sociedad en cómo ayudarlos social, emocional y asistencialmente, así como por parte de los familiares, quienes ignoran qué hacer y cómo atender a las personas adultas mayores cuando se encuentran con alteraciones en su salud física, orgánica, psicológica y mental (Jefatura del Gobierno del Distrito Federal, 2011).

Este trabajo se articula de la siguiente forma: primero se aborda el tema del envejecimiento y los problemas que enfrentan las personas mayores de 60 años, en particular las mujeres. Sucesivamente, se considera el marco institucional a nivel internacional en el cual, a pesar de algunos recientes avances, todavía, no hay un llamado explícito a este grupo etario enfatizando el rol del Gobierno del Distrito Federal (GDF) como un importante punto de referencia en cuanto al compromiso legal e institucional con las personas adultas mayores. La respuesta del GDF a las personas adultas mayores según sus derechos articula a través de la Secretaría de Desarrollo Social del Distrito Federal, de la cual depende el Instituto para la Atención de los Adultos Mayores en el Distrito Federal (IAAM-DF). En este tenor se indican los esfuerzos y los alcances de las acciones que el IAAM-DF realiza en relación a distintas necesidades de las personas adultas mayores. Finalmente, se presentan las conclusiones y los retos pendientes.

\section{EL ENVEJECIMIENTO}

El envejecimiento es un proceso natural que comienza desde el nacimiento del individuo, manifestándose a través de distintos cambios físicos y biológicos. Durante las etapas de la vida una persona necesita, adquiere y acumula capacidades, conocimientos y recursos que, además de permitirle gozar de un determinado nivel de vida, le serán de utilidad en las etapas sucesivas. En un primer momento estos elementos son proporcionados por los padres, y posteriormente es responsabilidad de la persona misma procurárselos, eventualmente recibiendo el apoyo por parte de la sociedad. No obstante, es esta última la que debería tomar una particular predominancia cuando la persona adulta mayor no está en grado de proveer por sí misma a sus necesidades.

Al envejecer, las capacidades de un individuo se modifican y su salud en particular, puede verse afectada; se observan cambios en sus actividades, intereses, prioridades, tipo y profundidad de las relaciones sociales. Adicionalmente, se reconoce que el envejecimiento es un proceso que se asocia con la pérdida gradual de las

1 En México, según la Ley de los Derechos de las Personas Adultas Mayores, y en la Ciudad de México de conformidad con lo dispuesto por el artículo 3 fracción I de la Ley de los Derechos de las Personas Adultas Mayores en el Distrito Federal, las personas adultas mayores son aquellas que tienen 60 años o más de edad que se encuentren domiciliadas o en tránsito en el territorio nacional. Aun cuando en otros países la denominación de estas personas pueda diferir (a menudo se hace referencia a ellos como a las personas de la tercera edad), al tratarse de normas de derecho positivo, el término personas adultas mayores se aplica en México, sin perjuicio para aquellas denominaciones con que puedan conocerse estas personas en alguna otra legislación. 
capacidades motrices y cognoscitivas; un mayor riesgo de discapacidad; la posibilidad de contraer enfermedades crónico-degenerativas como la diabetes, cardiovasculares, el cáncer y los trastornos de salud mental. Estos aspectos resultan ser una importante barrera para una plena y efectiva participación de las personas adultas mayores.

Desde una perspectiva de género, las mujeres con más de 65 años están más expuestas a padecer enfermedades crónicas que limitan su movilidad y capacidad para vivir sin necesidad de cuidados especiales. La investigación gerontológica explica este proceso mediante el concepto de "doble riesgo." Las mujeres se encuentran en una situación de mayor desventaja por el hecho de ser mayores y por su género (McMullin, 1996). A las mujeres mayores se les "(... ) tiende a percibir como más capaces de seguir cuidando tanto del hogar como de sí mismas (... )”, y así se “(... ) genera la presencia de muchas más mujeres mayores que necesitan cuidados y no los reciben, que hombres de ese grupo etario en la misma situación.” (Dolores, 2012: 331).

El carácter marcado por el género en el trabajo doméstico, la discriminación y los desequilibrios de poder dentro de la familia influyen en los factores de riesgo que siguen los cursos vitales de las mujeres y de los hombres. También se modifica la participación de las personas adultas mayores en la sociedad, de tal suerte que, dependiendo de la forma en que ésta se maneja tanto interna - por parte de la persona misma -, como externamente - por aquellos que la rodean, tengan o no una relación directa con ella -, esto influye sobre su calidad de vida y puede poner en riesgo la efectividad de sus derechos. En este sentido, la cercanía física de los familiares con las personas adultas mayores no garantiza que entre ellos se entable una comunicación verbal; de hecho, a pesar de la cercanía, los familiares no les dirigen la palabra o no las buscan (Jefatura del Gobierno del Distrito Federal, 2011).

En el 2011 el Secretario General de las Naciones Unidas examinó por primera vez los problemas y desafíos en el campo de los derechos humanos de las personas mayores en su Informe ante la Asamblea, sosteniendo que el problema más apremiante al que se enfrentan las personas mayores es la pobreza, materializada en la carencia de vivienda adecuada, la malnutrición, la falta de atención médica relacionada con las enfermedades crónicas, el limitado acceso al agua potable y al saneamiento, el costo prohibitivo de los medicamentos o del tratamiento médico y la inseguridad de los ingresos económicos (Naciones Unidas, 2011). Esta situación se recrudece para las mujeres al presentar una mayor dependencia económica de la pareja, historiales laborales irregulares que se asocian con menores pensiones al momento de la jubilación, conjuntamente con una mayor longevidad respecto a los hombres. En este caso, la carencia de ingresos se explica por el hecho de que las mujeres, durante la juventud y en la vida adulta, han tenido que dedicar más tiempo a las labores domésticas y a las tareas del cuidado de la familia, repercutiendo en una menor posibilidad de insertarse en el mercado laboral. Así, aunque las diferencias de género en materia de pobreza en los hogares son menos marcadas en la vejez, la intensidad de la pobreza resulta ser más aguda para las mujeres mayores, ya que los recursos acumulados a lo largo de su vida y con los que se enfrenta a la vejez son menores que los de los hombres. En el foro El adulto mayor en el Distrito Federal. Por una sociedad integral en el siglo XXI, organizado por la Asamblea Legislativa del Distrito Federal en 1997, Lozano (Jefatura de Gobierno del Distrito Federal, 2011) indicó que los adultos mayores pueden caer fácilmente en un ciclo enfermedad-pobreza, situación que está ligada a tres factores: I. el tipo de enfermedad, II. un bajo nivel de ingresos, y III. las dificultades para acceder a los servicios. Se desprende que para las personas adultas mayores la falta de recursos es una fuente de vulnerabilidad que implica una importante desventaja porque la autonomía económica está relacionada con la autonomía física y política (Montaño, 2012).

Desde un punto de vista de jure, independientemente de los recursos disponibles y de la edad, es evidente que los individuos mantienen intactos sus derechos como personas, pero no se puede no observar que de facto enfrentan obstáculos dictados por su condición y aceptación a nivel social. La solución de largo plazo no consiste en proporcionar ambientes o condiciones privilegiadas, sino de establecer una conciencia social dentro y fuera del ámbito familiar reconociendo que las personas adultas mayores no siempre pueden satisfacer sus necesidades de manera independiente y en la forma requerida. Mientras no exista suficiente conciencia social, mediada por los aspectos económicos y familiares que corresponden a cada caso, las personas adultas mayores están expuestas a tener que enfrentar varias dificultades sin apoyo. La extensión de este problema por su tamaño y alcance ha motivado que distintos actores a nivel privado (fundaciones, asociaciones civiles, OSCs, entre otros) y público (instituciones de gobierno), con los medios a su disposición, intenten ofrecer soluciones viables en el corto, mediano y largo plazo. Para tales efectos, aunque en el largo plazo se busca una mayor sensibilización por parte de la sociedad, en el corto plazo la presencia de acciones de corte asistencialista por parte de las instituciones públicas es indispensable para poder enfrentar aquellas necesidades básicas inmediatas, en particular cuando el apoyo por parte de los familiares es ausente. Es más, frente a la falta de apoyos por parte de las redes familiares, 
el gobierno tiene la responsabilidad y la obligación de velar por los intereses de los ciudadanos más desprotegidos.

\section{MARCO INSTITUCIONAL INTERNACIONAL}

Las personas adultas mayores ven reconocidos sus derechos en el marco jurídico internacional donde se indican los principios fundamentales de las personas en general, pero sin un énfasis explícito con respecto a la edad (Barahona Riera, 2012). No obstante, éstas son tuteladas desde la perspectiva de los derechos humanos y, en este sentido, las Naciones Unidas en el Pacto Internacional de Derechos Económicos, Sociales y Culturales (Naciones Unidas, 1966) - que debe y se ha interpretado de forma incluyente - señalan el derecho a la no discriminación en cuanto al empleo, acceso a la vivienda, cuidado de la salud, servicios sociales y alimentación (art.2), así como el derecho a ser tratados con dignidad, protección contra la violencia, el rechazo u otros tipos de abusos (art.12).

En vista del incremento de su participación a nivel mundial en las próximas décadas, tanto las condiciones como las necesidades de las personas adultas mayores han sido reconocidas a nivel internacional en la Segunda Asamblea Mundial sobre el Envejecimiento realizada en Madrid en el 2002. Consecuentemente, ha surgido una mayor conciencia que ha impulsado la "(...) tutela, protección y promoción de los derechos humanos de las personas mayores (...)" basados en el "(...) principio de igualdad y no discriminación de la vejez (...)" (Acle Mautones, 2012: 61). De acuerdo al principio de no discriminación plasmado en el Pacto Internacional de Derechos Económicos, Sociales y Culturales (Naciones Unidas, 1966, art.2), es una responsabilidad de los Estados el garantizar los derechos (también) de las personas adultas mayores, en términos legales y reales. De esta forma, se ha sugerido que los Estados deberían ver como una obligación el "(...) ampliar la cobertura de las pensiones mediante sistemas no contributivos y proveer pensiones alimentarias para las personas mayores sin recursos." (Barahona Riera, 2012: 237).

Aun cuando estas acciones sean justificadas desde un punto de vista social, se tuvo que esperar hasta el 2011 para un pronunciamiento explícito en cuanto a la necesidad de enfrentar la problemática relativa a los derechos de las personas mayores, evidenciando las distintas dimensiones, así como los principales obstáculos que enfrentan (Naciones Unidas, 2011).

\section{POLÍTICAS DIRIGIDAS A LAS PERSONAS ADULTAS MAYORES}

\section{Avances en América Latina}

Las normas que se refieren expresamente a la protección de las personas adultas mayores son relativamente recientes en los países de América Latina al promulgarse y aplicarse a fines de la década de 1980.

Debido a que la calidad de vida en la vejez depende de los progresos que se logran en las tres áreas prioritarias: I. las personas de edad y el desarrollo, II. el fomento de la salud, y III. la creación de entornos propicios y favorables (Pugliese, 2010), una de las limitaciones más importantes que se presentan en esta región para el seguimiento de la situación de las personas mayores, es la falta de análisis de información sobre estos temas fundamentales que guardan una estrecha relación con el bienestar.

Para los adultos mayores, uno de los elementos que más incide en su bienestar se asocia con lo económico, ligado a su vez con la calificación de la fuerza laboral. Para ello se considera que los niveles de calificaciones básicas que posee la población de edad avanzada, como por ejemplo los escasos niveles de alfabetización y capacidad de cálculo, propician la informalidad y la inestabilidad laboral, así como una baja remuneración (Paz, 2010). La búsqueda de opciones laborales por parte de las personas adultas mayores ocurre cuando quieren sentirse útiles y perciben estar en grado de poder desempeñar las actividades o no pueden mantener el nivel económico que tenían en los años previos ya sea porque no tienen derecho a una pensión suficientemente elevada, por no haber acumulado suficientes activos a lo largo de la vida, cuyas rentas les permitan reducir la presión económica o, en algunos casos, para seguir manteniendo a las generaciones más jóvenes (Villagómez, 2013).

De esta manera, y sobre todo en la edad avanzada, en caso de enfrentar una situación económica prohibitiva, las alternativas que quedan a disposición son: I. el abandono y la pobreza, II. compartir el domicilio con algún hijo o hija, a veces sufragando parte de los gastos, o III. accesar a algún asilo público o privado, según las posibilidades económicas de los descendientes de la persona adulta mayor.

De acuerdo con los relatos recopilados por Villagómez (2013), resulta evidente la necesidad de proporcionar alternativas laborales a las personas adultas mayores, pero éstas deben de estar coordinadas adentro de una estrategia de mayor alcance y profundidad (Viscusi, 1979). Por ejemplo, las personas deben comenzar a planear 
su retiro y ahorrar para la jubilación, en caso de tener acceso a un plan de jubilación contributivo, desde la etapa productiva y alcanzar esta meta en el largo plazo. Eventualmente, si las personas no tuvieran la posibilidad de tener acceso a una jubilación, deberían tratar de ahorrar de acuerdo al nivel de vida que planean alcanzar una vez que ya no se desempeñen en el mercado laboral, teniendo en cuenta que los patrones de gasto varían con la edad (OECD, 2005).

Para mitigar los problemas económicos que se derivan de la precariedad laboral de las personas adultas mayores, en América Latina se han implementado iniciativas que buscan colmar las brechas de conocimientos y/o habilidades, y agilizando su colocación en el mercado laboral. Ejemplo de ello es la oferta de capacitación para el empleo en Chile, Colombia, El Salvador, Honduras, Panamá, Puerto Rico y Uruguay; o el desarrollo de bancos de datos e información para poder facilitar el conseguir empleo a las personas mayores (en El Salvador, México y Puerto Rico). Estas acciones son necesarias frente a la discriminación laboral de la cual son objeto las personas adultas mayores por razones de edad, pero más importante aun es el ejemplo que ofreció Panamá en 2007, cuando la Asamblea Nacional aprobó que los trabajadores del sector públicos con 75 años cumplidos pudieran seguir desempeñándose en este sector (Paz, 2010).

\section{Avances en México y en el Distrito Federal}

En México, en 1979 el Gobierno Federal creó el Instituto Nacional de la Senectud (INSEN) el cual, durante las siguientes dos décadas llevó a cabo tareas médico-asistenciales en beneficio de las personas adultas mayores (Villagómez, 2013). De esta forma, la política social implementada por el Estado Mexicano no tenía mayor impacto, limitándose a la formación de clubes, talleres de artesanías/manualidades, y a la creación de asilos, gestionando, a través de la firma de convenios con el sector privado, descuentos para reducir la carga económica que tenía que soportar esta población en la compra de algunos artículos de primera necesidad como, por ejemplo, los medicamentos.

Reconociendo que las personas adultas mayores eran "invisibles" a los programas, acciones sociales y servicios del Estado, las autoridades de la Ciudad de México plantearon la necesidad de abatir los importantes rezagos en las áreas de salud, económica, educativa y social. La búsqueda de una sociedad incluyente, que respete los derechos de este sector de la población, se asocia con una percepción diferente de la vejez y del envejecimiento y requiere de un enfoque integral y coordinado de atención.

En la Ciudad de México, el GDF constituye un importante punto de referencia por cuanto concierne a los derechos de las personas adultas mayores. El 7 de marzo de 2000 en la Gaceta Oficial del Distrito Federal se publicó la Ley de los Derechos de las Personas Adultos Mayores en el Distrito Federal (Asamblea Legislativa del Distrito Federal, 2000b) la cual refrenda el derecho de los adultos mayores de vivir una vida con calidad. En particular, de forma consistente con la visión de Capital Social establecida por el actual Jefe de Gobierno y cuya responsabilidad recae principalmente en la Secretaría de Desarrollo Social del Distrito Federal, el GDF provee a las personas adultas mayores una atención integral: alimentación, vivienda, recreación, medio ambiente saludable, educación, pensión alimentaria (a los adultos mayores de 68 años), servicios médicos y medicamentos gratuitos, crédito para vivienda, descuentos en los impuestos de predial y agua, pasaje gratuito en autobuses RTP, STC Metro, Trolebús, Tren Ligero y Metrobús. Debido a que las personas adultas mayores pueden estar sujetas a situaciones de violencia, a través de distintos acuerdos con otras instancias del GDF, se han creado las Unidades de Atención a la Violencia Familiar que proporcionan orientación y asesoría para atender y solucionar aquellas situaciones de maltrato físico, económico, psicológico o sexual.

La Ley de los Derechos de las Personas Adultas Mayores en el Distrito Federal señala que las personas adultas mayores (art.5) tienen derecho a no ser discriminadas, a una vida libre de violencia, a ser protegidos contra toda forma de explotación y a recibir protección por parte de su familia como por parte de las autoridades. Asimismo, se reconoce la importancia del entorno y de las relaciones sociales y familiares buscando que vivan de forma digna y decorosa manteniendo contacto con la familia cuando no estén con ella.

En relación a la procuración de justicia, tienen el derecho a recibir el apoyo por parte de las instancias competentes, contar con asesoría jurídica gratuita y con un representante legal, en caso de ser necesario, para buscar proteger su patrimonio. También, se garantiza que puedan satisfacer las necesidades de salud, alimentación y el acceso a los programas de asistencia social y laboral para poder gozar de una mejor calidad de vida.

La Comisión Nacional de los Derechos Humanos (CNDH, 2012) reconoce estos derechos que constituyen el fundamento jurídico para proporcionar la atención integral que actualmente ofrece el GDF. Al garantizar estos 
derechos se busca que las personas adultas mayores puedan tener una vida propia y que se desempeñen de forma independiente.

A nivel federal, un importante avance en cuanto a la satisfacción de las necesidades de las personas adultas mayores ocurrió a comienzo de la década pasada cuando el INSEN se convirtió en el Instituto Nacional de Adultos en Plenitud (INAPLEN) el cual estaba coordinado por el órgano rector de la vigilancia del respeto de los derechos a los adultos mayores, la Secretaría de Desarrollo Social (SEDESOL). El 25 de junio de 2002 se publicó en el Diario Oficial de la Federación la Ley de los Derechos de las Personas Adultas Mayores (Congreso de la Unión, 2002). Con esta Ley se establecieron las bases y disposiciones para garantizar el ejercicio de los derechos de las personas adultas mayores mediante la regulación de: I. la política pública nacional para la observancia de los derechos de las personas adultas mayores, II. los principios, objetivos, programas, responsabilidades e instrumentos que la administración pública federal, las entidades federativas y los municipios deberán observar en la planeación y aplicación de la política pública nacional, y III. el Instituto Nacional de las Personas Adultas Mayores (INAPAM), que reemplazó el INAPLEN. Esta Ley se reformó por última vez el 9 de abril del 2012 en los artículos 38 y 40 al introducir los lineamientos de conformación del Consejo Directivo y del funcionamiento del INAPAM.

\section{Personas adultas mayores en el Distrito Federal}

En México, la pirámide de población para el 2010 mostraba una clara prevalencia de la población joven de edad inferior a los 20 años, a pesar de una menor participación de los niños entre o y 4 años. A medida que aumenta la edad, de forma simétrica de acuerdo al género, la participación disminuye paulatinamente y vuelve a incrementarse a partir de los 75 años (Figura 1). A pesar de que este ensanchamiento es esperado, debido a que se considera un rango de edad mayor respecto a los demás, se observa una menor participación a nivel nacional de este segmento etario en comparación al observado en la Ciudad de México (Figura 2).

FIGURA 1

PIRÁMIDE DE POBLACIÓN MÉXICO 2010

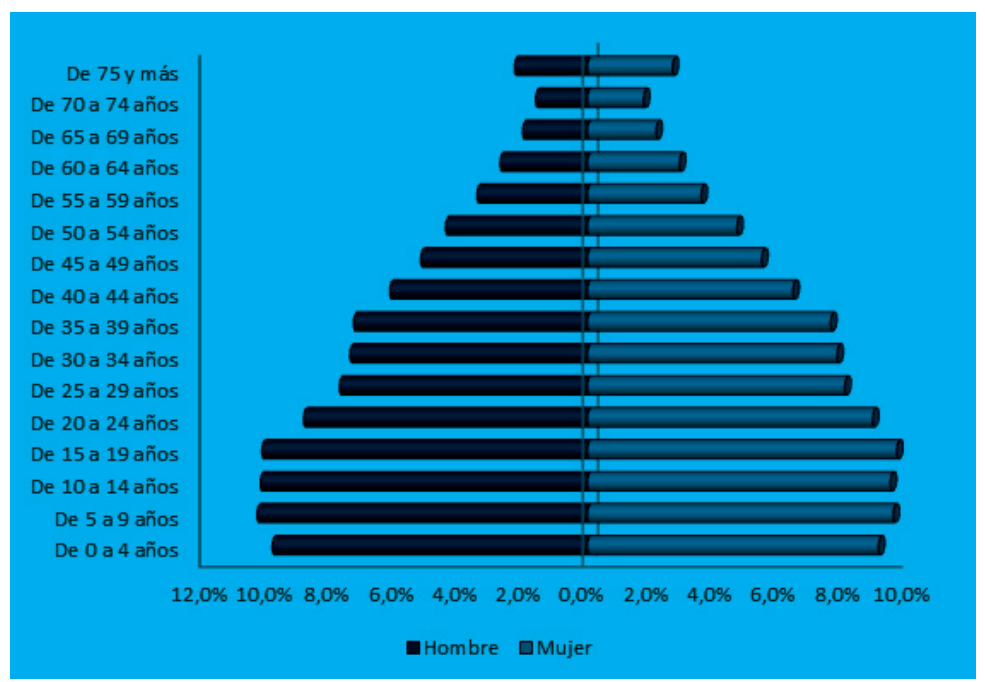

Fuente: Elaboración propia con datos de INEGI, Censo de Población y Vivienda, 2010.

En la Ciudad de México, también conocida como Distrito Federal al ser sede de los poderes federales de los Estados Unidos Mexicanos, la pirámide demográfica para el 2010 mostraba una menor participación de los estratos de población más jóvenes y un ensanchamiento en los estratos entre los 20 y los 39 años. De igual forma que en la figura 1, al rebasar los 75 años de edad ambos géneros, pero sobre todo las mujeres, aumentan su participación. 
FIGURA 2

PIRÁMIDE DE POBLACIÓN DISTRITO FEDERAL 2010

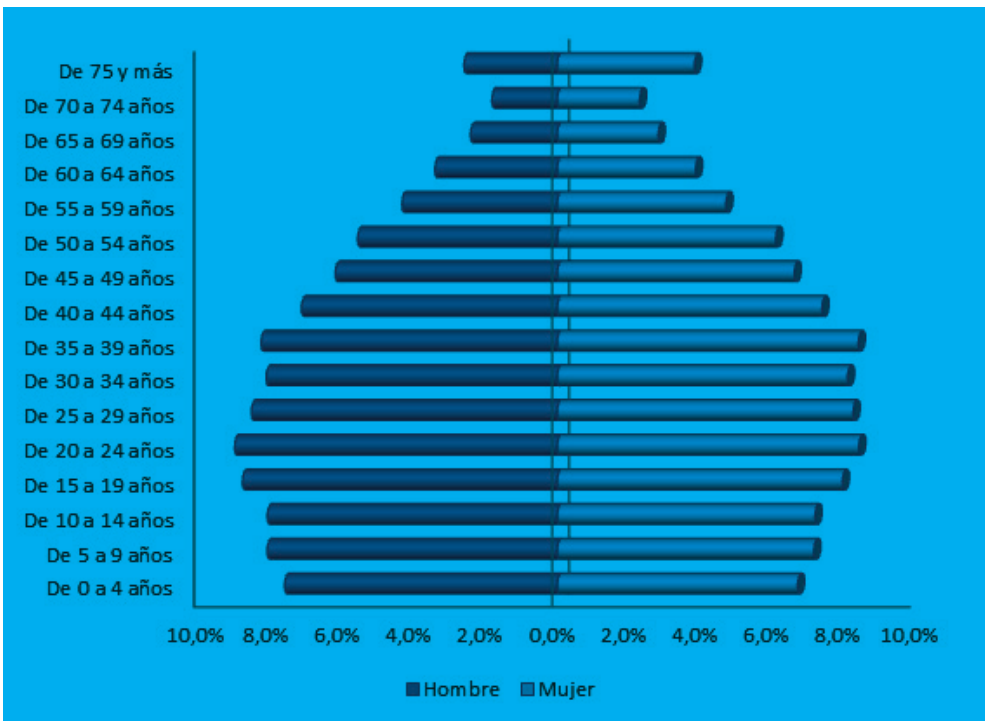

Fuente: Elaboración propia con datos de INEGI, Censo de Población y Vivienda, 2010.

En el marco de la celebración del Día Internacional de las Personas de Edad (1 de octubre) el INEGI compila estadísticas con temas relacionados a empleo, salud, mortalidad y problemas de los adultos mayores para cada uno de los estados de la República Mexicana. Para el Distrito Federal, la concentración y evolución de la población se ha caracterizado por la transición demográfica hacia el envejecimiento. El INEGI (2011a) reporta que en 2010 en el Distrito Federal habitaban 1,003,648 personas de 60 años y más, siendo la entidad a nivel nacional con el mayor porcentaje de población envejecida (11.3\%).

TABLA 1

DISTRIBUCIÓN PORCENTUAL DE LA POBLACIÓN 1950-2030 EN LA CIUDAD DE MÉXICO

\begin{tabular}{lccccccccc}
\hline Grupos de edad (años) & 1950 & 1960 & 1970 & 1980 & 1990 & 2000 & 2010 & 2020 & 2030 \\
\hline o a 14 & 35.9 & 41 & 41.5 & 37 & 30.5 & 26.1 & 21.9 & 20.5 & 18.8 \\
15 a 59 & 58.7 & 53.5 & 53.2 & 57.3 & 62 & 63.9 & 64.6 & 63.9 & 60.8 \\
6o y más & 5.2 & 5.2 & 5.3 & 5.7 & 7.1 & 8.5 & 11.3 & 15.6 & 20.4 \\
No especificado & 0.2 & 0.3 & 0 & 0 & 0.4 & 1.5 & 2.2 & 0 & 0 \\
\hline Total & 100 & 100 & 100 & 100 & 100 & 100 & 100 & 100 & 100 \\
\hline Índice de envejecimiento & 14.5 & 12.7 & 12.8 & 15.4 & 23.3 & 32.6 & 51.6 & 76.1 & 108.5 \\
\hline
\end{tabular}

Fuente: CONAPO, Proyecciones de la Población, 2010-2030. INEGI, Censo de Población y Vivienda, 2010.

De acuerdo con la información demográfica proporcionada por el Instituto Nacional de Estadística y Geografía (INEGI) y el Consejo Nacional de Población (CONAPO), las proyecciones para el 2020 y el 2030 anticipan que en los próximos quince años la proporción de personas mayores de 60 años rebasará a los menores de 15 años (Tabla 1).

Por esta razón, el índice de envejecimiento (la relación entre la población de 60 y más años respecto a la población menor de 15 años) se incrementará paulatinamente, lo cual representa un llamado para proyectar y seguir atendiendo a las necesidades de este grupo etario. Un rasgo distintivo del envejecimiento de la población ya evidenciado en las pirámides poblacionales a nivel nacional y para la Ciudad de México, es el predominio femenino como consecuencia de una mayor esperanza de vida de las mujeres respecto a los hombres.

En las siguientes décadas se espera que la participación de las personas de edad de 0 a 14 años se reduzca del $21.9 \%$ (2010) al 20.5\% (2020) para alcanzar el 18.8\% en el 2030, mientras que los mayores de 60 años aumentarían el 11.3\% (2010) al 15.6\% (2020) y finalmente al 20.4\% (2030). En el 2010, la delegación² Benito Juárez presentaba el

2 La Ciudad de México está dividida en 16 delegaciones y cada una es un órgano político-administrativo para el funcionamiento del GDF (http://www.df.gob.mx/index.php/delegaciones). 
mayor índice de envejecimiento, 115 adultos mayores por cada 100 niños, veinte años antes había registrado un índice de 55 adultos mayores por cada 100 niños. Al igual que la Benito Juárez, las delegaciones Miguel Hidalgo y Coyoacán registraron unos índices de envejecimiento del 78.3 y 76.6\% respectivamente. El menor valor se observó para la delegación Milpa Alta, 26 adultos mayores por cada 100 niños, resultado de la conformación de la estructura por edad de esta delegación, en donde los grupos de edades jóvenes crecen a un ritmo mayor en comparación con otras delegaciones (INEGI, 2011a). En la figura 3 se reporta el rango del índice de envejecimiento por cada delegación de la Ciudad de México.

\section{FIGURA 3}

\section{ÍNDICE DE ENVEJECIMIENTO POR DELEGACIÓN, 2010}

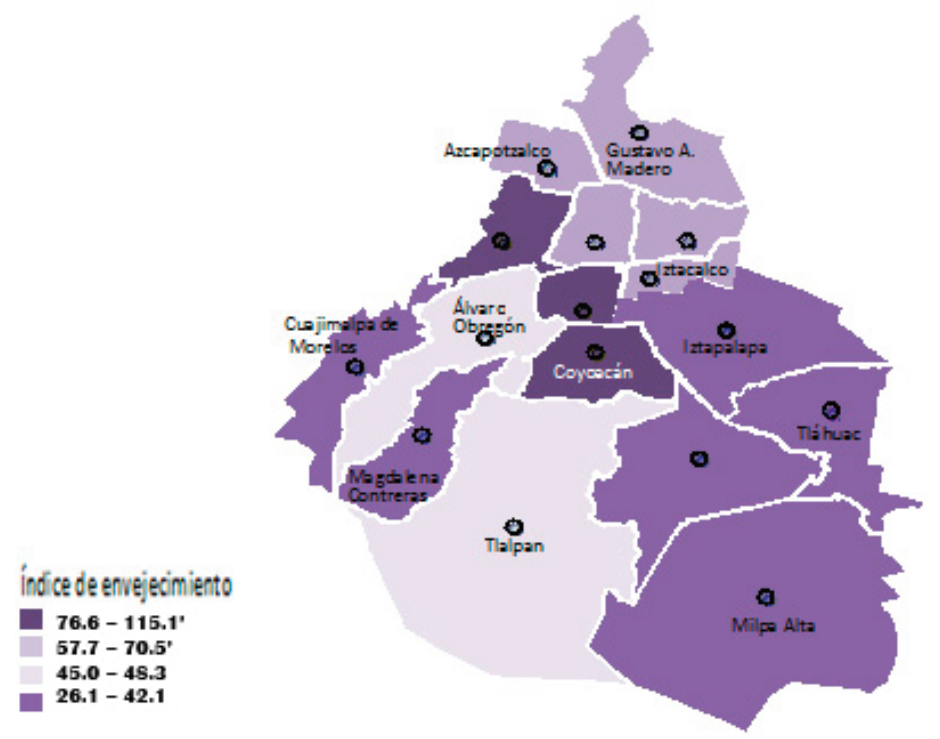

Fuente: INEGI, Censo de Población y Vivienda, 2010.

El INEGI (2013a) reporta también el índice masculinidad (número de hombres por cada cien mujeres) de la población adulta mayor en el Distrito Federal; mientras que el índice en 2010 era de 73 hombres por cada 100 mujeres se espera que para el 2030 aumente levemente hasta 74 hombres por cada 100 mujeres.

En cuanto a vulnerabilidad y pobreza, la población mayor de 60 años en el Distrito Federal se encuentra en una mejor situación respecto a la que reside en el resto del país (Tabla 2), resultado de la presencia de los servicios a disposición de la población en esta ciudad y de las políticas que han sido implementadas por parte del GDF. No obstante estos porcentajes, en las delegaciones existen todavía diferencias que justifican la necesidad de seguir realizando acciones e implementar programas focalizados hacia los grupos de población más vulnerable.

Los resultados destacan que en el Distrito Federal se ha avanzado en cuanto a la solución de las carencias sociales y a la solución de la pobreza; en este sentido, la vulnerabilidad por ingresos en la Ciudad de México resulta ser 6.5 veces más baja que a nivel nacional.

TABLA 2

PORCENTAJE DE LA POBLACIÓN DE 60 AÑOS Y MÁS POR SITUACIÓN DE POBREZA Y VULNERABILIDAD, 2012

\begin{tabular}{lcc}
\hline \multirow{2}{*}{ Indicadores } & \multicolumn{2}{c}{ Total } \\
\cline { 2 - 3 } & Distrito Federal & México \\
\hline Población vulnerable por carencias sociales & 28.7 & 72 \\
Población vulnerable por ingresos & 7.4 & 49.4 \\
Población en situación de pobreza & 18.7 & 43.2 \\
Población no pobre y no vulnerable & 45.3 & 21.8 \\
\hline
\end{tabular}

Fuente: Elaboración propia con datos del INEGI (2013a, 2013b). 


\section{EL INSTITUTO PARA LA ATENCIÓN DE LOS ADULTOS MAYORES EN EL DISTRITO FEDERAL}

El GDF mediante la creación del Instituto para la Atención de los Adultos Mayores en el Distrito Federal (IAAM-DF) ha reconocido la importancia de realizar acciones estructuradas, incluyentes y orientadas a promover políticas públicas, implementando programas de atención integral, impulsando el ejercicio pleno de sus derechos, y fomentando una cultura del envejecimiento activo. Es decir, mejorar la calidad de vida de las personas adultas mayores que residen en la Ciudad de México.

EI IAAM-DF se creó por Decreto del Jefe de Gobierno, publicado en la Gaceta Oficial del Distrito Federal el 29 de junio de 2007, presentando antecedentes en el "Programa de Apoyo Alimentario, Atención Médica y Medicamentes Gratuitos para Adultos Mayores de 70 Años Residentes en el Distrito Federal” en febrero de 2001 y en la aprobación de la entonces Ley que establece el Derecho a la Pensión Alimentaria para los Adultos Mayores de 70 Años Residentes en el Distrito Federal en 2003 (IAAM-DF, 2014a).

Actualmente el IAAM-DF tiene a su cargo el Programa de Pensión Alimentaria para Adultos Mayores de 68 Años Residentes en el Distrito Federal y opera varias acciones; entre las cuales destacan:

1. Visitas Médicas Domiciliarias para los derechohabientes de la Pensión Alimentaria,

2. Visitas Gerontológicas,

3. Agencia Especializada para la Atención a Adultos Mayores Víctimas de Violencia Familiar de la Procuraduría General de Justicia,

4. Turismo Social "Hacer turismo es hacer vida”,

5. Escuela de Mayores, y

6. Mañanas de película.

\section{Programa de Pensión Alimentaria para Adultos Mayores de 68 Años Residentes en el Distrito Federal}

El Programa de Pensión Alimentaria ha sido implementado por el Gobierno del Distrito Federal desde el 20013. Gracias a su contribución en la mejora de la calidad de vida de los adultos mayores, se aprobó en 2003 la Ley por la que se estableció el Derecho a la Pensión Alimentaria para Adultos Mayores de 70 Años Residentes en el Distrito Federal, la cual se actualizó en 2008 ampliando el margen de edad a 68 años. Este programa tiene como rectora a la Ley de Desarrollo Social del Distrito Federal (Asamblea Legislativa del Distrito Federal, 2000a) ya que debe cumplir el artículo 4 donde se señalan los 12 principios de la política de desarrollo social4.

El Objetivo General del Programa de Pensión Alimentaria es “Otorgar una seguridad económica básica, garantizando el pleno ejercicio del derecho a la pensión alimentaria a toda persona adulta mayor de 68 años que cumpla con los requisitos establecidos en la Ley que Establece el Derecho a la Pensión Alimentaria para Adultos Mayores de 68 Años Residentes en el Distrito Federal y su Reglamento. Esta pensión económica tiene como finalidad atenuar las desigualdades sociales que enfrenta este grupo de población5." (IAAM-DF, 2014a).

Los requisitos que se solicitan para que un adulto mayor sea derechohabiente del Programa son:

1. Tener 68 años o más al momento de solicitar su inscripción al padrón de derechohabientes de la Pensión Alimentaria.

2. Radicar permanentemente en el Distrito Federal, con una antigüedad mínima de tres años de residencia al momento de la solicitud de la pensión, lo que deberá acreditar a través de cualquiera de los medios reconocidos por la Ley y el Reglamento.

El medio utilizado para materializar este beneficio es una tarjeta electrónica en la que se depositan

3 A partir de 2014 el INAPAM, coordinado por la SEDESOL, ha implementado el Programa "Pensión para Adultos Mayores" que busca contribuir a la ampliación de los esquemas de seguridad social universal para las personas adultas mayores. La población objetivo del programa son las personas de 65 años de edad en adelante mexicanos por nacimiento o con un mínimo de veinticinco años de residencia en el país, que no reciban pensión mayor a 1,092 pesos mensuales por concepto de jubilación o pensión de tipo contributivo. El apoyo económico consiste de 580 pesos mensuales con una dispersión del recurso de 1,160 pesos cada dos meses. Este programa se basa en los 10 principios de la Política de Desarrollo Social que señala el artículo 3 de la Ley General de Desarrollo Social (Congreso de la Unión, 2004).

4 Estos principios son: I. Universalidad, II. Igualdad, III. Equidad de Género, IV. Equidad Social, V. Justicia Distributiva, VI. Diversidad, VII. Integralidad, VIII. Territorialidad, IX. Exigibilidad, X. Participación, XI. Transparencia, y XII. Efectividad.

5 Al haberse elevado a rango de Ley el derecho de recibir la pensión alimentaria, las personas que la reciben ya no se consideran beneficiarias sino derechohabientes del programa. 
mensualmente recursos económicos para la adquisición de productos en establecimientos comerciales del Distrito Federal como tiendas de autoservicio, restaurantes, farmacias, mercados públicos, etc., y que a diciembre del 2013 sumaban 5,296. No permite disponer de efectivo para brindar seguridad al pensionado en su uso y con ella se garantiza la atención de los requerimientos individuales objetivos y subjetivos de cada adulto mayor, permitiéndoles incluso el ahorro.

Durante el 2013 el Programa brindó una Pensión Alimentaria Mensual de 971.40 pesos por derechohabiente, que representa un aumento del 3.89\% con respecto al 2012. Para el año 2014, el Jefe de Gobierno del Distrito Federal anunció el aumento de la pensión a 1,009.35 pesos mensuales efectivos a partir de febrero del 2014 y con efecto retroactivo para el pago de enero del mismo año. El monto a percibir permite acceder a una canasta básica compuesta por productos que integran la pirámide alimenticia para una mejor nutrición tales como: pollo, pescado, carne, leguminosas, verduras, leche, frutas, tortilla, y azúcar, entre otros. Para poder mantener el poder de compra de los derechohabientes del Programa debido al incremento en precios de los productos de la canasta, la pensión mensual se ha incrementado anualmente entre 20 y 40 pesos (Figura 4).

El mayor beneficio de una transferencia monetaria respecto a una en especie está ampliamente justificado desde un punto de vista económico sobre el bienestar de los derechohabientes debido a que estos estarían en grado de adquirir lo que necesitan con los recursos proporcionados (Viscusi, 1979).

El número de derechohabientes con el que inició el Programa en 2001 fue de 150,000 personas. Actualmente alcanza los 480,000 derechohabientes y hasta finales del 2013, han sido beneficiadas 916,964 personas adultas mayores. Aumentando año con año su presupuesto alrededor del 7 y 10\%, el Programa de Pensión Alimentaria recibió un presupuesto en 2013 de 5,459,000 millones de pesos.

FIGURA 4

MONTO EN PESOS DE LA PENSIÓN ALIMENTARIA 2001-2014.

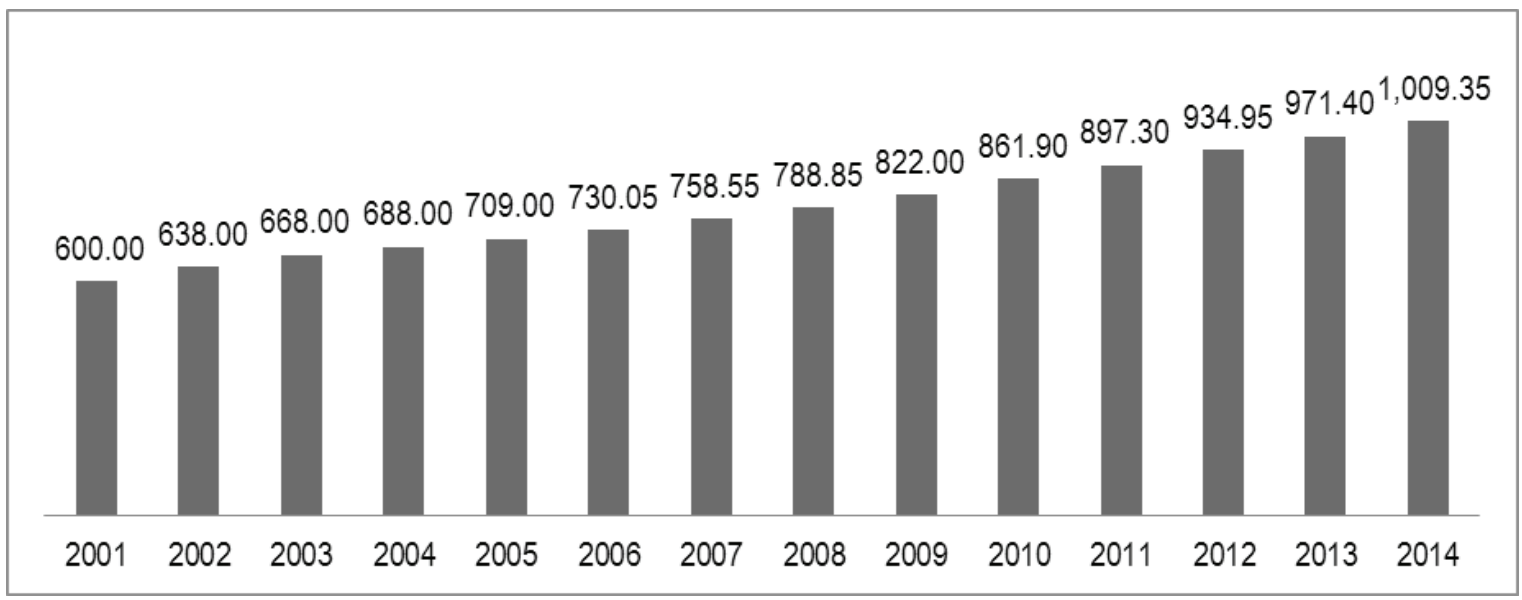

Fuente: Elaboración propia con datos del Instituto para la Atención de los Adultos Mayores del Distrito Federal (2014).

Las evaluaciones realizadas al Programa revelan que la atención prestada a las personas adultas mayores por parte del IAAM-DF ha sido particularmente bien recibida ya que el $80 \%$ de la población derechohabiente la ponderó entre 9 y 10 (valor máximo). También sobresale que los indicadores del Programa están debidamente soportados con información institucional y que guardan relación con los objetivos del mismo siendo diseñados para cuantificar los resultados y avances esperados. La constante evaluación al Programa le ha permitido transitar de un primer enfoque bajo el criterio de focalización territorial hasta convertirse en una Pensión Universal Ciudadana (IAAM-DF, 2013).

El Programa contribuye al logro de la equidad e integración social, a partir de que el adulto mayor en el núcleo familiar se encuentra como un actor activo con capacidad para sustentar sus necesidades básicas.

\section{Acciones complementarias en beneficio de las personas adultas mayores}

En el foro organizado por la Asamblea Legislativa del Distrito Federal en 1997 se expusieron distintos tipos de maltrato a los cuales podían estar sujetas las personas adultas mayores: físico, psicológico, sexual, económico y social. El maltrato físico, cuando implica lesiones y existe denuncia, es relativamente fácil de detectar; por lo 
contrario, el maltrato psicológico, que se manifiesta a través del "retraimiento social, depresión, desordenes de ansiedad o conductuales.", es más difícil de detectar, pues a las agresiones verbales y actitudes impositivas hacia las personas adultas mayores se pueden sumar otras igual de violentas, como ignorarlos o tratarlos de manera infantil, lo cual, apuntaron los especialistas, "es una forma de aversión al envejecimiento" (Jefatura del Gobierno del Distrito Federal, 2011:28).

Las personas adultas mayores son también víctimas de agresiones económicas, como el despojo de sus bienes, el uso ilegal de su pensión o de su tarjeta bancaria; a veces los familiares no utilizan el dinero para su bienestar desviando sus recursos y evitando sufragar los gastos para su cuidado, así como presionándolo a distribuir o heredar sus bienes en vida.

En relación al maltrato social algunos ejemplos consisten en no cederles el paso en las calles, ser impacientes con ellos, segregarlos e ignorarlos en los espacios públicos.

Con el fin de beneficiar a las personas adultas mayores en cuanto a atención médica, seguridad, educación y esparcimiento, el IAAM-DF ejecuta acciones que, de forma integral y coordinada con otras dependencias del GDF, contribuyen a alcanzar un mejor nivel de bienestar también en estas áreas.

\section{Visitas Médicas Domiciliarias para los derechohabientes de la Pensión Alimentaria}

La desigualdad en el acceso a los servicios de salud y el desamparo son más evidentes para las personas adultas mayores, por lo que se requiere cubrir con nuevos beneficios a ese grupo, teniendo como objetivos principales la protección y el mejoramiento de las condiciones individuales de salud, la disminución de la desigualdad ante la enfermedad y la elevación de la calidad de vida. El IAAM-DF frente a la necesidad de atender de manera integral a las personas adultas mayores ha puesto en marcha las Visitas Médicas Domiciliarias para Adultos Mayores.

Durante estas visitas se proporciona atención médica a los derechohabientes del Programa Pensión Alimentaria y su objetivo es realizar la Valoración Geriátrica Integral a todo adulto mayor atendido para conocer las condiciones en las que vive. Esto permite percibir si el adulto mayor sufre de violencia física, emocional y/o sexual ya sea por parte de familiares u otras personas de confianza, porque por su misma naturaleza resulta difícil documentarla o monitorearla adecuadamente, ya que muchas personas mayores prefieren no denunciar o no tienen los mecanismos idóneos para hacerlo.

Las visitas ofrecen un beneficio inherente para las personas adultas mayores adscritas al Programa de Pensión Alimentaria, lo que constituye su único requisito; favorece la participación del cuidador y/o de la familia al capacitarlos para la atención y asistencia de las personas adultas mayores.

Las actividades que realiza el médico en la visita domiciliaria tienen varios objetivos entre los cuales destacan:

1. Llevar a cabo una Valoración Geriátrica Integral.

2. Determinar, a través de una valoración geronto-geriátrica, si la persona adulta mayor requiere atención médica en el domicilio.

3. Priorizar los problemas y las intervenciones para determinar el mejor nivel de atención médica que necesita la persona adulta mayor.

4. Realizar actividades de prevención y promoción de la salud.

5. Programar visitas médicas domiciliarias para poder proporcionar un seguimiento de la atención integral.

\section{Visitas Gerontológicas}

La Coordinación de Gerontología del IAAM-DF promueve la participación solidaria y organizada de los distintos actores sociales involucrados en la atención de las personas adultas mayores, como lo son la familia, las personas que integran su comunidad y las dependencias del GDF. Para realizar una valoración integral se realizan visitas domiciliarias gerontológicas y conjuntas (véase sección anterior, numeral 2) en atención al adulto mayor y, a partir de los resultados de la visita, se ponen a consideración de la persona adulta mayor y su familia las estrategias a seguir para entablar una relación de empatía para que los interesados se sientan comprendidos y respaldados. La atención gerontológica también se brinda por vía telefónica, en persona en las instalaciones del IAAM-DF o por Internet. Entre diciembre 2012 y diciembre 2013 se realizaron 971 visitas domiciliarias y se atendieron 2,383 solicitudes telefónicas o por internet (IAAM-DF, 2014b). 
Adicionalmente, la Coordinación organiza cursos de capacitación y sensibilización en las dependencias del GDF y otras instituciones para promover una cultura de la vejez, fomentando que las personas adultas mayores reciban un trato digno y adecuado en la atención de sus necesidades. Para ello, el personal de la Coordinación se capacita constantemente en temas de derechos humanos, género, lenguaje no sexista, y gerontología social comunitaria, participando, como representante del IAAM-DF, en los consejos, comités y comisiones en otras dependencias del GDF para que los programas y líneas de acción que se implementen incluyan contenidos gerontológicos y procedimientos de atención especial dirigidos a las personas adultas mayores.

\section{Agencia Especializada para la Atención a Adultos Mayores Víctimas de Violencia Familiar de la Procuraduría General de Justicia}

De acuerdo con la Encuesta Nacional de la Dinámica de las Relaciones en los Hogares (INEGI, 2011b) se identifican cuatro tipos de violencia contra las mujeres: emocional, económica, física y sexual. En el Distrito Federal, de las mujeres unidas y alguna vez unidas de 60 años y más, el 54.3\% declaró haber sufrido algún tipo de violencia por parte de su pareja o expareja a lo largo de la relación.

Debido a los problemas de abandono y violencia familiar y con el objetivo de proporcionar una atención integral a las personas adultas mayores, en el 2010 se suscribió un acuerdo entre la Procuraduría General de Justicia del Distrito Federal (PGJDF) y el IAAM-DF. A partir de este acuerdo, en el mismo año se realizaron distintas acciones dirigidas a solucionar esta problemática tales como: la creación de la Agencia Especializada para la Atención a Adultos Mayores Víctimas de Violencia Familiar de la Procuraduría General de Justicia, proporcionar un mejor acceso telefónico y virtual para las personas adultas mayores a los servicios del Ministerio Público, y la integración de la Red por la Defensa de los Derechos de los Adultos Mayores, entre otras. Asimismo, para reducir la incidencia de aquellos episodios que por su vulnerabilidad ven involucradas a las personas adultas mayores se instituyó un programa para fomentar la cultura de la denuncia de los derechos humanos entre las personas adultas mayores de la Ciudad de México. A la par, el IAAM-DF ofrece un servicio de atención telefónica operado por el Consejo Ciudadano de Seguridad Pública y Procuración de Justicia denominada "Línea Plateada" (5533 5533). Este servicio se brinda las $24 \mathrm{hrs}$. los 365 días del año.

Además de contribuir a la seguridad de las personas adultas mayores, algunas de las actividades que realiza el IAAM-DF de manera integral, buscan también fomentar la integración social y cultural.

\section{Turismo Social "Hacer turismo es hacer vida"}

De gran relevancia es el convenio firmado entre la Secretaría de Desarrollo Social del Distrito Federal, a través del IAAM-DF, y la Secretaría de Turismo el 12 de marzo del 2013 para arrancar "Hacer turismo es hacer vida".

El objetivo consiste en "promover el derecho a la educación, recreación y cultura, con un enfoque intergeneracional, a favor del envejecimiento activo, la cohesión social y familiar; lo anterior a través de recorridos turísticos locales y nacionales". Como parte de la estrategia que busca materializar este derecho, se organizan y realizan viajes de bajo costo para las personas adultas mayores. El IAAM-DF y la Secretaría de Turismo promueven, con empresas y prestadores de servicios, paquetes turísticos preferentemente en beneficio de los derechohabientes de la Tarjeta de la Pensión Alimentaria para Adultos Mayores.

Desde marzo hasta diciembre del 2013, se han realizado 320 viajes (198 viajes locales y 122 foráneos) beneficiando a 13 mil 457 adultos mayores. A nivel local los destinos son los centros históricos de las diferentes delegaciones del Distrito Federal, mientras que los 122 viajes foráneos, para 5 mil 532 adultos mayores, tuvieron destinos tales como: Acapulco, Hidalgo, Ixtapan de la Sal, Oaxtepec, Puebla, Veracruz, y Querétaro, entre otros.

Las personas adultas mayores viajan acompañadas por personal médico, enlaces y educadoras comunitarias del IAAM-DF, de la Secretaría de Desarrollo Social y guías turísticos de la Secretaría de Turismo.

\section{Escuela de Mayores}

Esta acción, implementada desde diciembre 2010, hasta el mes de abril de 2014 ha sido respaldada a través de la creación de 123 Escuelas para Mayores, las cuales tienen el objetivo de "Fomentar la dignificación de las personas mayores creando espacios para la construcción colectiva de conocimiento útil, en el que se comparte lo que se sabe, se aprende lo que se necesita y se plantea la transformación como forma de evidenciar y concretizar los aprendizajes".

La Escuela de Mayores es un espacio educativo que busca desarrollar e integrar colectivos de adultos mayores para que compartan sus experiencias y sabiduría. Asimismo, se conscientiza sobre las necesidades de formación 
de los adultos mayores, por lo que las educadoras han diagnosticado, mediante el diálogo acerca de las demandas de sus integrantes, las actividades de su interés y que se realizan: manualidades, bordado, teatro, música, yoga, computación, natación, talleres de lectura, etc.

Estas escuelas sesionan una vez a la semana orientadas y acompañadas por una educadora del IAAM-DF la cual, siendo objeto de una constante capacitación, cuenta con una participación promedio de 30 adultos mayores por sesión.

\section{Mañanas de película}

Con esta acción, que arrancó en el mes de febrero del 2014, se “promueve con las empresas exhibidoras de películas la donación de entradas gratis al cine y tiene como objetivo general el garantizar el derecho a la cultura y recreación de las Personas Adultas Mayores derechohabientes del Programa Pensión Alimentaria para Adultos Mayores de 68 Años, Residentes en el Distrito Federal".

Las personas adultas mayores pueden asistir al cine gratis dos veces al mes y en su tarjeta de la Pensión Alimentaria se les abona una cortesía cada quince días (cortesías no acumulables).

Al llegar al cine, los beneficiarios deben presentar su tarjeta de la Pensión Alimentaria y una identificación oficial para hacer válida su cortesía de lunes a viernes en funciones programadas antes de las 16:0ohrs.

\section{CONCLUSIONES}

El proporcionar una respuesta a las necesidades de las personas adultas mayores es una tarea compleja que incluye la prevención y detección anticipada de éstas para poder administrar una solución eficaz; consecuentemente, de ninguna forma la atención puede, ni debe, limitarse al 'ejercicio del presupuesto'. Actualmente, el Programa de Pensión Alimentaria y las acciones que encausa el IAAM-DF aminoran los problemas relacionados con la pobreza alimentaria y de patrimonio, atienden necesidades específicas de salud considerando la edad de los derechohabientes, fomentan el esparcimiento y la integración social. Otras necesidades, como el acceso a medicamentos gratuitos y transportes están garantizadas a través de convenios con otras dependencias del GDF que, de forma coordinada, velan por una mayor inclusión social. Todos estos esfuerzos están también alineados con que las personas adultas mayores sigan gozando de un envejecimiento exitoso al buscar mantener su funcionalidad a través de la realización de distintas actividades y conservando de un buen estado nutricional y de salud (Martínez Arronte, 2004).

No obstante, las necesidades de las personas adultas mayores varían y, entre otros factores, la urgencia de satisfacerlas está en función del contexto en el cual se originan. Por esta razón la búsqueda de soluciones integrales, eficaces y eficientes es una tarea que se realiza de forma continua con el apoyo y la colaboración de distintas dependencias del GDF. En este sentido, el IAAM-DF preside la Secretaría Técnica Adjunta del Consejo Asesor cuyo fin último es "favorecer la plena integración y desarrollo de las personas adultas mayores" (IAAM-DF, 2014a). Desde un punto de vista proactivo, es conveniente que el Instituto establezca un sistema de detección, análisis y seguimiento de las nuevas necesidades de las personas adultas mayores para poder determinar su prioridad y la(s) forma(s) más idónea(s) para su solución, contar con recursos y estructura para tales efectos. De esta forma, se podrán aprovechar economías de escala y de alcance tanto en la cobertura del Programa como de las acciones que actualmente se están llevando a cabo, y que son constantemente orientadas a satisfacer las necesidades de las personas adultas mayores.

Aunque el GDF, a través de la Secretaría de Desarrollo Social del Distrito Federal y el IAAM-DF, se ha dado a la tarea de ofrecer un conjunto de programas y acciones que buscan de forma integrada la inclusión social y un mejor nivel de vida para las personas adultas mayores, se reconoce que estos esfuerzos deberán aumentar con base en el continuo incremento de la población objetivo debido a la universalidad del Programa de Pensión Alimentaria. En particular, esta situación es el resultado de dos factores: el primero es una componente inercial ligada a la estructura demográfica de la Ciudad de México, mientras que el segundo - más difícil de cuantificar - consiste en el aumento de la demanda de la pensión alimentaria para adultos mayores debido a la migración hacia el DF desde otras entidades federativas. Para ello, el IAAM-DF, a pesar de contar con 1,300 educadoras, 71 capacitadores o enlaces, 20 coordinadores regionales, 8 gerontólogos y 45 médicos geriatras que constituyen el personal operativo del Instituto, en los próximos años deberá buscar una ampliación tanto presupuestal como del número de colaboradores, para poder seguir proporcionando un seguimiento cercano a las personas adultas mayores. 


\section{Agradecimientos}

Se agradece a la Dirección General del IAAM-DF la información proporcionada para la preparación de este documento.

\section{BIBLIOGRAFÍA}

Acle Mautones, M. (2012), "El principio de igualdad y no discriminación en la vejez y la introducción de la perspectiva de edad", en S. Huenchuan, ed., Los derechos de las personas mayores en el siglo XXI: situación, experiencias y desafíos: 59-74. Santiago de Chile: Comisión Económica para América Latina y el Caribe (CEPAL) y Gobierno de la Ciudad de México.

Asamblea Legislativa del Distrito Federal. (2000a), “Ley de Desarrollo Social para el Distrito Federal”. En línea: <http://cgservicios.df.gob.mx/prontuario/vigente/d1875.pdf> (Consulta: 18 septiembre 2014)

Asamblea Legislativa del Distrito Federal. (2000b), "Ley de los derechos de las personas adultas mayores en el Distrito Federal”. En línea: <http://cgservicios.df.gob.mx/prontuario/vigente/r20604.pdf> (Consulta: 20 octubre 2014)

Barahona Riera, R. (2012), "Nivel de vida adecuado, derechos humanos y envejecimiento", en S. Huenchuan, ed., Los derechos de las personas mayores en el siglo XXI: situación, experiencias y desafíos: 229-242. Santiago de Chile: Comisión Económica para América Latina y el Caribe (CEPAL) y Gobierno de la Ciudad de México.

Comisión Nacional de los Derechos Humanos [CNDH]. (2012), "Derechos de los adultos mayores". En línea: <http://www.cndh.org.mx/sites/all/fuentes/documentos/cartillas/9\%20cartilla\%20Derechos\%20adultos\%20 mayores.pdf> (Consulta: 17 septiembre 2014)

Congreso de la Unión. (2002), “Ley de los derechos de las personas adultas mayores”. En línea: <http:// cgservicios.df.gob.mx/prontuario/vigente/r275901.pdf> (Consulta: 22 octubre 2014)

Congreso de la Unión. (2004), “Ley General de Desarrollo Social”. En línea: <http://cgservicios.df.gob.mx/ prontuario/vigente/dr35302.pdf> (Consulta: 16 septiembre 2014)

Dolores, M. (2012), “La vida en femenino. ¿Construyendo fortaleza o fragilidad para la vejez?”, en S. Huenchuan, ed., Los derechos de las personas mayores en el siglo XXI: situación, experiencias y desafíos: 325-347. Santiago de Chile: Comisión Económica para América Latina y el Caribe (CEPAL) y Gobierno de la Ciudad de México.

Instituto Nacional de Geografía y Estadística [INEGI]. (2010), “Censo de Población y Vivienda, 2010. Tabulados del cuestionario básico”. México, D.F.: Instituto Nacional de Geografía y Estadística (INEGI).

(2011a), “Estadísticas a Propósito del Día Internacional de las personas de edad. Datos para el Distrito Federal”. México, D.F.: Instituto Nacional de Geografía y Estadística (INEGI).

(2011b), “Encuesta Nacional sobre la Dinámica de las Relaciones en los Hogares”. Base de datos. México, D.F.: Instituto Nacional de Geografía y Estadística (INEGI).

(2013a), “Estadísticas a Propósito del Día Internacional de las personas de edad. Datos para el Distrito Federal”. México, D.F.: Instituto Nacional de Geografía y Estadística (INEGI).

(2013b), "Estadísticas a Propósito del Día Internacional de las personas de edad. Datos Nacionales". México, D.F.: Instituto Nacional de Geografía y Estadística (INEGI).

Instituto para la Atención de los Adultos Mayores en el Distrito Federal [IAAM-DF]. (2013), "Evaluación Interna del Programa de Pensión Alimentaria para Adultos Mayores de 68 Años”. México, D.F.: Instituto para la Atención de los Adultos Mayores en el Distrito Federal (IAAM-DF). enero 2014)

(2014a), Página oficial del IAAM-DF. En línea: <http://www.adultomayor.df.gob.mx/> (Consulta: 12

(2014b), “Numeralia IAAM 2013”. México, D.F.: Instituto para la Atención de los Adultos Mayores en el Distrito Federal.

Jefatura del Gobierno del Distrito Federal. (2011), “Nueva vida para nuestros mayores”. México, D.F.: Gobierno del Distrito Federal. 
Martínez Arronte, F. (2004), “Salud y autocuidado”, en N. Asili, ed., Vida plena en la vejez: 141-150. México, D.F.: Ed. Pax México.

McMullin, J. (1996), “Teoría de las relaciones de edad y género”, en S. Arber y J. Ginn, ed., Relaciones de Género y Envejecimiento. Enfoque Sociológico: 54-70. Madrid: Narcea.

Montaño, S. (2012), “Las mujeres mayores y el envejecimiento con dignidad en América Latina”, en S. Huenchuan, ed., Los derechos de las personas mayores en el siglo XXI: situación, experiencias y desafíos: $311-323$. Santiago de Chile: Comisión Económica para América Latina y el Caribe (CEPAL) y Gobierno de la Ciudad de México.

Naciones Unidas. (1966), “Pacto Internacional de Derechos Económicos, Sociales y Culturales”, Resolución 2200 (XXI) de la Asamblea General, $21^{\circ}$ período de sesiones. Nueva York.

_ (2011), "Derechos humanos de personas mayores: Resumen del Informe del Secretario General para la Asamblea (A/66/173)”. En línea: <www.ohchr.org/Documents/Issues/OlderPersons/Resumen_informe_personas_ mayores2011.doc> (Consulta: 6 enero 2014).

Organisation for Economic Cooperation and Development [OECD]. (2005), "Improving Financial Literacy Analysis of Issues and Policies". Paris: Organisation for Economic Cooperation and Development Publications.

Paz, J. (2010). “Envejecimiento y empleo en América Latina y el Caribe”. Sector del Empleo Documento de Trabajo Núm. 56. Ginebra: Organización Internacional del Trabajo.

Pugliese, L. (2010), “Marco normativo para los derechos de las personas adultas mayores”. En línea: <http:// www.bps.gub.uy/innovaportal/file/1516/1/marco_normativo_para_los_derechos_de_las_personas_adultas_ mayores._I._pugliese.pdf> (Consulta: 20 octubre 2014). Yucatán.

Villagómez, G. (2013), “No es pecado envejecer”. México, D.F.: Ed. M.A. Porrúa y Universidad Autónoma de

Viscusi, K. (1979), “Welfare of the Elderly - An Economic Analysis and Policy Prescription”. New York: John Wiley \& Sons, Inc. 